\title{
Differential susceptibility to the tracheal mite Acarapis woodi between Apis cerana and Apis mellifera
}

\author{
Yoshiko SaKamoto $^{1}$, Taro Maeda ${ }^{2}$, Mikio YoshiYama ${ }^{3}$, Jeffery S. Pettis ${ }^{4}$ \\ ${ }^{1}$ National Institute for Environmental Studies, 16-2 Onogawa, Tsukuba, Ibaraki 305-8506, Japan \\ ${ }^{2}$ Institute of Agrobiological Sciences, NARO, 1-2 Ohwashi, Tsukuba, Ibaraki 305-0851, Japan \\ ${ }^{3}$ Institute of Livestock and Grassland Science, NARO, 2 Ikenodai, Tsukuba, Ibaraki 305-0901, Japan \\ ${ }^{4}$ USDA-ARS Bee Research Laboratory BLDG. 306 BARC-E, Beltsville, MD 20705, USA
}

Received 14 January 2016 - Revised 25 May 2016 - Accepted 4 July 2016

\begin{abstract}
In Japanese honey bees Apis cerana japonica, infestations of the tracheal mite Acarapis woodi have spread rapidly over the mainland of Japan, causing damage and the collapse of colonies. Meanwhile, infestations by mites in Apis mellifera have hardly been observed in Japan. In this study, we assessed and compared the susceptibility of the two species, A. cerana and A. mellifera, using an inoculation assay. We found that migrating female mites entered the tracheae of more newly emerged bees in both species but more frequently in A. cerana than in A. mellifera. Hence, the higher susceptibility in A. cerana is proposed as a factor causing the explosive epidemic of tracheal mites in only A. cerana in Japan. Moreover, we compared grooming behaviors between the two bee species using an observation assay as a preliminary experiment, although the bees were not exposed to the presence of tracheal mites. From these observations, the frequency of autogrooming (self-grooming) on the thorax in A. cerana was lower than that in A. mellifera. The difference in susceptibility to the mite between these two species may be due to the difference in grooming behavior frequency.
\end{abstract}

\section{Asian honey bee / European honey bee / Acarapis woodi / susceptibility / grooming behavior}

\section{INTRODUCTION}

The tracheal mite Acarapis woodi, which was first associated with a condition that caused considerable colony mortality in Apis mellifera on the Isle of Wight, England, in the early 1900s (Rennie 1921), has spread all over the world with the exception of Sweden, Norway, Denmark, and Australia (Sammataro et al. 2000). Both larval and adult mites feed on bee hemolymph in the tracheal tubes of adult bees (Pettis and Wilson 1996), and mated females leave the trachea and move to new callow bees to begin a new infestation (Sammataro and Needham 1996). Mites are more successful in

Corresponding author: Y. Sakamoto, sakamoto.yoshiko@nies.go.jp

Manuscript editor: Peter Rosenkranz moving to new bees at night when the adult bees are not as active (Pettis et al. 1992). Heavy mite infestation results in colony losses by decreasing longevity (Bailey and Lee 1959), low honey production (Eischen et al. 1989), and the inability to thermoregulate in cool climates (Eischen 1987; McMullan and Brown 2009), due to damage of the tracheal system. Major sources of the spread of tracheal mites between colonies could be the drifting of infested bees to adjacent hives and swarming (Eckert 1961).

In 2010, infestations by the tracheal mite A. woodi were first recorded in Apis cerana japonica in Japan (Ministry of Agriculture, Forestry and Fisheries 2011). In Japan, there are two Apis species, the Japanese honey bee A. cerana japonica which is a native subspecies existing only in Japan and the western honey bee $A$. mellifera which is a non-native species now found virtually worldwide (Ruttner 1988). Mite infestations have spread 
rapidly across a wide range of mainland Japan in A. cerana japonica (Maeda 2015), causing heavy damage and the collapse of colonies during winter (Maeda and Sakamoto 2016). Meanwhile, mite infestations in A. mellifera have hardly been observed in Japan (Kojima et al. 2011; Maeda 2015), despite the fact that foragers of A. mellifera (Sasaki 1999) and $A$. cerana (our observation, unpublished) enter each other's hives to steal honey, thus mixing species. In recent years in A. mellifera, mite infections are not epidemic or problematic, even in places once heavily mite-infested (Wilson et al. 1997).

So why does only A. cerana japonica suffer from the mites in Japan? To understand this would be useful information for the conservation of A. cerana japonica. However, at this time the factors are not clear and could be multifactorial. One potential factor is the use of miticides in hives of A. mellifera. A natural ectoparasitic mite, Varroa destructor, of A. cerana has shifted to A. mellifera and is present on A. mellifera almost worldwide (Anderson and Trueman 2000; Solignac et al. 2005). In Japan, two miticides, fluvalinate and amitraz, are used only in hives of A. mellifera which have no effective defense against the Varroa mite. However, it is not clear how effective these Varroa mite treatments are for reducing tracheal mite infestations (Scott-Dupree and Otis 1992), even though there have been some positive reports in lab experiments (Eischen et al. 1986; Pettis et al. 1988). Another possibility is genetic resistance to the mite in A. mellifera. Some genetic strains of A. mellifera resist damaging infestations of the mites (Danka 2001), and the resistance is linked to allogrooming and autogrooming behaviors (Pettis and Pankiw 1998; Danka and Villa 2003). Hence, A. mellifera has likely evolved grooming behaviors against the mite, which at least partially explains the reduced mite damage in A. mellifera . Mite infestation mechanisms in A. cerana need to be studied, and a comparison of mite susceptibility between A. cerana and A. mellifera is needed.

In this article, we assessed and compared the susceptibility of the two Apis species to tracheal mites. The purpose of experiment 1 was to determine age susceptibility of each bee species over the first 4 days of adult life using an inoculation assay. Experiment 2 also used an inoculation assay to compare the susceptibility of 0 -day-old bees (newly emerged bees, known to be most susceptible in A. mellifera) between the two species. In addition, we conducted an experiment to compare the grooming behavior between the two species by using an observation assay (experiment 3 ).

\section{MATERIALS AND METHODS}

\subsection{Honey bee colonies}

We utilized bee colonies of $A$. cerana japonica and A. mellifera, which were kept at the National Institutes for Environmental Study (NIES), Institute of Agrobiological Sciences, NARO (NIAS), Institute of Livestock and Grassland Science, NARO (NIRGS), and private apiaries in Tsukuba City, Ibaraki Prefecture, Japan. We did not administer any Varroa treatments at least a half year before starting the experiments in all A. mellifera colonies. All laboratory assays were conducted at NIES, and a field assay was conducted at NIAS. Experiments 1 and 2 were undertaken in November 2014. Experiment 3 was conducted in August 2015.

\subsection{Preparation of host and target bees for inoculation experiments 1 and 2}

We conducted inoculation assays by putting intact live bees and mite-infested bees together under laboratory and field conditions. We refer to the mite-infested bees as "host" bees and the intact bees to be tested as "target" bees (Gary and Page 1987). We collected foragers from a heavily infested bee hive of A. cerana japonica at the hive entrance and used them as host bees. We had confirmed a $100 \%$ infestation rate in the foragers 1 month earlier. To obtain target bees, combs containing emerging bees from each colony were taken to the laboratory and kept in cages in an incubator to allow for adult bee emergence $\left(35^{\circ} \mathrm{C}\right.$, $55 \% \mathrm{RH})$. Emerged bees ( $0-24 \mathrm{~h}$ old) were removed from the combs and marked with paint (Paint Marker, Mitsubishi, Japan) on their abdomen to distinguish age and colony source. All bees for these experiments, except host bees in a hive experiment, were narcotized by $\mathrm{CO}_{2}$ for $30 \mathrm{~s}$ before starting, to avoid rejection and to mix the bees thoroughly, and a preliminary experiment confirmed that there was no bias of proportional distribution between bee species. Host and target bees from the same colony were not used within an experiment. 


\subsection{Experiment 1-susceptibility of bees less than four days old}

The purpose of experiment 1 was to assess the agerelated susceptibility of each bee species from 0 to 3 days of age, not to compare the level of susceptibility between bee species. The mite ordinarily rarely enters the tracheae of the bees older than 4 days of age (Lee 1963; Gary et al. 1989; Phelan et al. 1991). Target bees of four different ages, <24, 24-48, 48-72, and 72-96 h (or $0,1,2$, and 3 days of age) were prepared for each assay of bee species. Numbers of target bees of A. cerana and A. mellifera were $150(34,36,40$, and 40 with $0-3$ days of age) and $150(39,40,37$, and 34 with $0-3$ days of age), respectively. One- to 3 -day-old target bees were maintained with sugar water $(50 \%$ $v / v$ ) in a dark incubator at $32{ }^{\circ} \mathrm{C}$ and $55 \% \mathrm{RH}$ until used in the experiment. On November 10, the target bees of A. cerana and A. mellifera were separately put into cages $(15 \mathrm{~cm} \times 15 \mathrm{~cm} \times 15 \mathrm{~cm}$; BugDorm, MegaView Science, Taichung, Taiwan). The cages for A. cerana and A. mellifera are referred to as cages I and II and were maintained under dark conditions at $32{ }^{\circ} \mathrm{C}$ and $55 \%$ RH. We put 47 and 52 individuals in cages I and II as host bees in conjunction with adding the target bees, respectively. The bees were provided ad libitum with sugar water $(50 \% \mathrm{v} / \mathrm{v})$ via a plastic petri dish $(2 \mathrm{~cm} \times 9 \mathrm{~cm}$ diameter) with three holes $(8 \mathrm{~mm}$ diameter) on the bottom of the cage. A piece of comb $(7 \mathrm{~cm} \times 4 \mathrm{~cm} \times 3 \mathrm{~cm})$ was put on a wire and wood stand $(6 \mathrm{~cm} \times 12 \mathrm{~cm} \times 4 \mathrm{~cm})$ after freezing at $-20{ }^{\circ} \mathrm{C}$ for $24 \mathrm{~h}$ to kill wax moths. Figure 1 shows the experimental layout with the cage. We choose 7 days as the test period based on the evidence that the first adult mites to mature are the males after 11-12 days (Pettis and Wilson 1996). Hence, after 7 days, the only adult mites present in the target bees are the founding females. The dead bees were removed from the cage, segregated, and counted. The live bees were put in a freezer and kept at $-20{ }^{\circ} \mathrm{C}$ for $24 \mathrm{~h}$, and then bees were segregated into host or target bee categories and counted. The target bees were also stored at $-20{ }^{\circ} \mathrm{C}$ to await dissection.

\subsection{Experiment 2-interspecies comparison of susceptibility}

Inoculation assays were conducted twice in the same cages as in experiment $1(15 \mathrm{~cm} \times 15 \mathrm{~cm} \times 15 \mathrm{~cm}$; BugDorm, MegaView Science, Taichung, Taiwan) and once in a Langstroth hive under field conditions. In this experiment, we used 0-day-old intact bees of both A. cerana and A. mellifera together as target bees to eliminate any unexpected differences among experimental setups. We started the cage experiment (cages I and II) on November 10 and 12, respectively. The total number of introduced host and target bees is shown in Table I. Four and three colonies of A. cerana and A. mellifera, respectively, were used to supply target bees. The experimental procedure of cage inoculation assay was the same as in experiment 1. For the hive inoculation assay, we introduce 0 -day-old target bees of A. cerana and A. mellifera into a heavily infested hive in which outside workers were shown to be $100 \%$ infested. The hive assay was performed beginning on November 13. The hive was estimated to have more than 3000 workers during that period. After 7 days, the target bees were removed from the hive using forceps. The storage method for bees was the same as in experiment 1 above.

\subsection{Dissection technique}

The bees were dissected using a modification to the classic technique of removing the head and thoracic collar as described by Lorenzen and Gary (1986). The prothoracic tracheae were carefully removed without crushing the end and placed on a double-sided tape placed on a glass slide. Using a stereomicroscope (M205C; Leica Microsystems $\mathrm{GmbH}$, Wetzlar, Germany) at $\times 60-160$ magnification, each trachea was opened using a microneedle. The number of adult mites of each sex, along with the number of larvae and eggs, was counted and recorded. This method mainly follows Mcmullan and Brown (2005).

\subsection{Experiment 3-interspecies comparison in the frequency of grooming behaviors}

An observation glass hive $(47 \mathrm{~cm} \times 27 \mathrm{~cm} \times 5 \mathrm{~cm})$ was used to examine the frequency of grooming behaviors. A comb $(45 \mathrm{~cm} \times 23 \mathrm{~cm})$ was used and partly filled by $74 \%$ fructose and sucrose solution. Newly emerged bees $<24$-h old (73 workers from an A. cerana colony and 152, 270, and 242 workers from three A. mellifera colonies) were prepared and marked for this experiment. Additionally, approximately 500 workers obtained from another colony of A. mellifera were put into the 

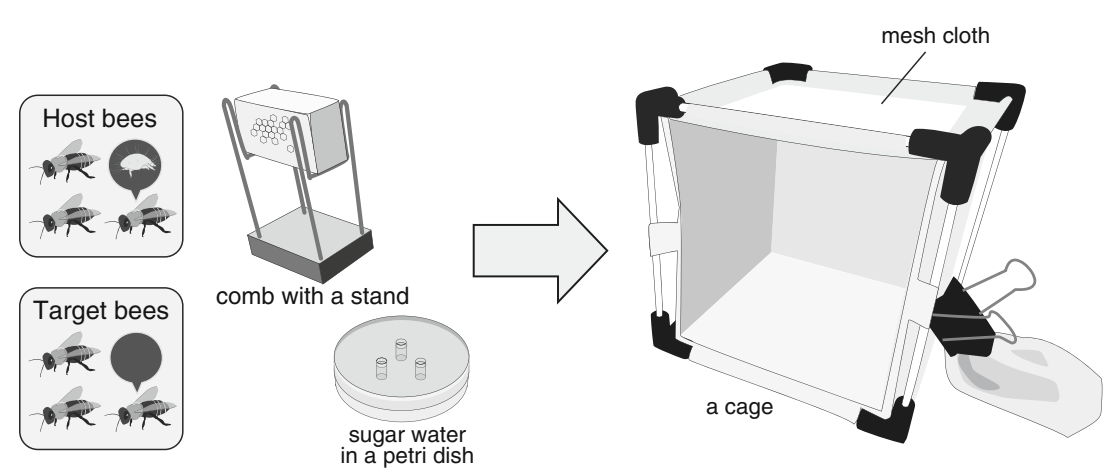

Figure 1. The experimental layout with a cage. Host bees, target bees, comb on the stand, and sugar water in a plastic petri dish within a cage of mesh cloth were maintained under dark conditions at $32{ }^{\circ} \mathrm{C}$ and $55 \% \mathrm{RH}$ for 7 days. The petri dish lid has three holes ( $8 \mathrm{~mm}$ diameter) attached to a vinyl chloride tube ( $15 \mathrm{~mm}$ length) to prevent bees from falling into the sugar water.

same case to fill the comb. All bees for this experiment were narcotized by $\mathrm{CO}_{2}$ for $30 \mathrm{~s}$ before starting to avoid rejection between colonies in the observation hive. The observation hive was maintained at $25{ }^{\circ} \mathrm{C}$ in a laboratory and covered with a cloth except during the observations. The observations were made between 8:00 and 18:00 on the next 2 days after setting bees in the observation hive. We observed randomly selected 10 marked bees in succession. This sequential observation which consisted of 10 individuals was referred to as a "scan," thus one scan needed 1 min $(6 \mathrm{~s} \times 10$ bees $)$. Each bee was observed for seconds, and its behavior was categorized into four categories: (1) allogrooming (nestmate cleaning), (2) being allogroomed, (3) autogrooming (self-cleaning), and (4) no grooming. We also recorded the body parts being groomed and which legs were used to groom. In this study, we defined a grooming to the dorsolateral thorax anteriorly by a middle leg as "thorax-autogrooming." We conducted five scans for each colony with more than 3-min intervals between scans. These sequential five scans were called a "set." A total of seven sets of observations were conducted in each colony for 2 days with more than 1.5-h intervals for each individual set. We conducted 35 and 105 scans of behavior observation in A. cerana and A. mellifera in total, respectively.

\subsection{Statistical analyses}

All analyses were conducted using the statistical software R 3.1.1 (R Development Core Team 2014).

\section{RESULTS}

\subsection{Experiment 1 -susceptibility of bees less than four days old}

The total number of live target bees of A. cerana and A. mellifera were 53 (survival rate $35 \%)$ and $135(90 \%)$, respectively. Figure 2 clearly showed that both number of entering adult mites and infestation rate of trachea were higher in younger emerged bees irrespective of bee species. The declines in mite infestation rates with increasing bee age between the two species were similar.

\subsection{Experiment 2 -interspecies comparison of susceptibility}

The results are shown in Table I. The rate of infested bees in A. cerana was significantly higher than that in A. mellifera in cage I and II assays but not in the case of the hive assay (Table I). The number of mites per trachea in A. cerana is greater than that in A. mellifera in all inoculation assays (Figure 3). There was no significant intraspecific difference of the number of mites per trachea in intraspecies among colonies in each assay ( $p>0.05$, Mann-Whitney or Steel-Dwass test) except for A. mellifera in the hive assay ( $p<0.05$, Steel-Dwass test). Overall, the ratio of larvae to eggs in A. cerana was significantly greater than that of $A$. mellifera in 
Sakamoto Y. et al.

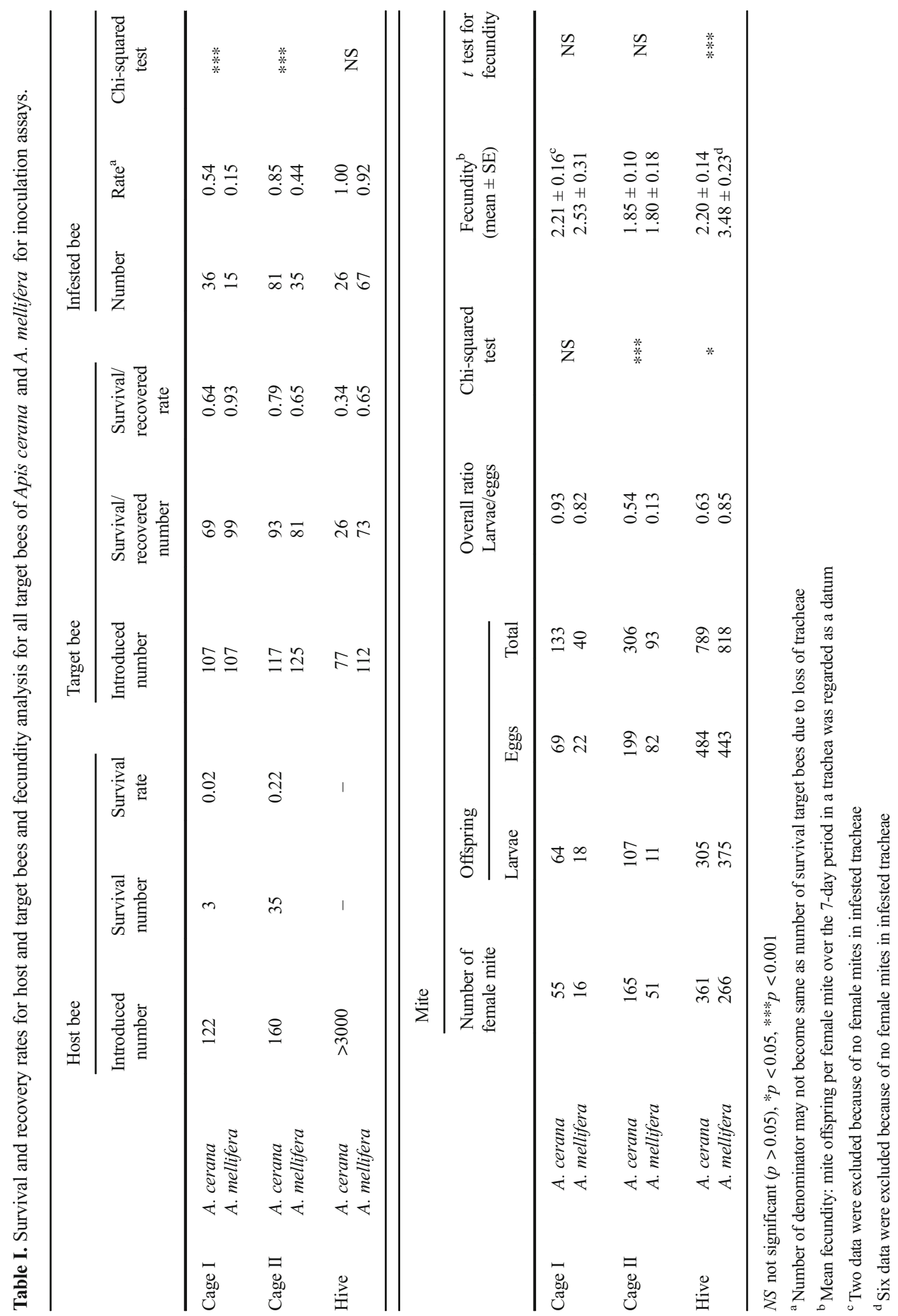




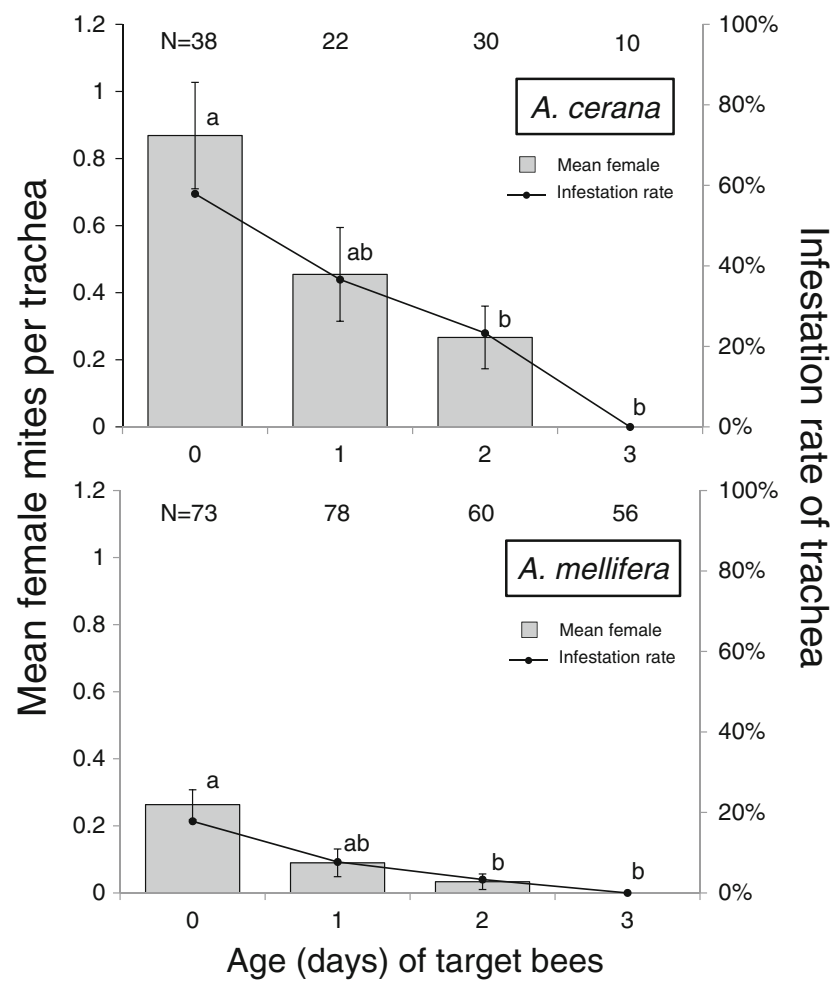

Figure 2. Mean adult tracheal mites Acarapis woodi found in the large thoracic tracheae of two Apis species, A. cerana (upper) and A. mellifera (lower), aged 0-3 days in inoculation assay after 7 days. Columns with the same letter in each honey bee species are not significantly different at $5 \%$ levels by Steel-Dwass test. The data are expressed in means \pm SE to show the decline clearly although not parametric. Secondary axis shows the infestation rate of trachea (line chart).
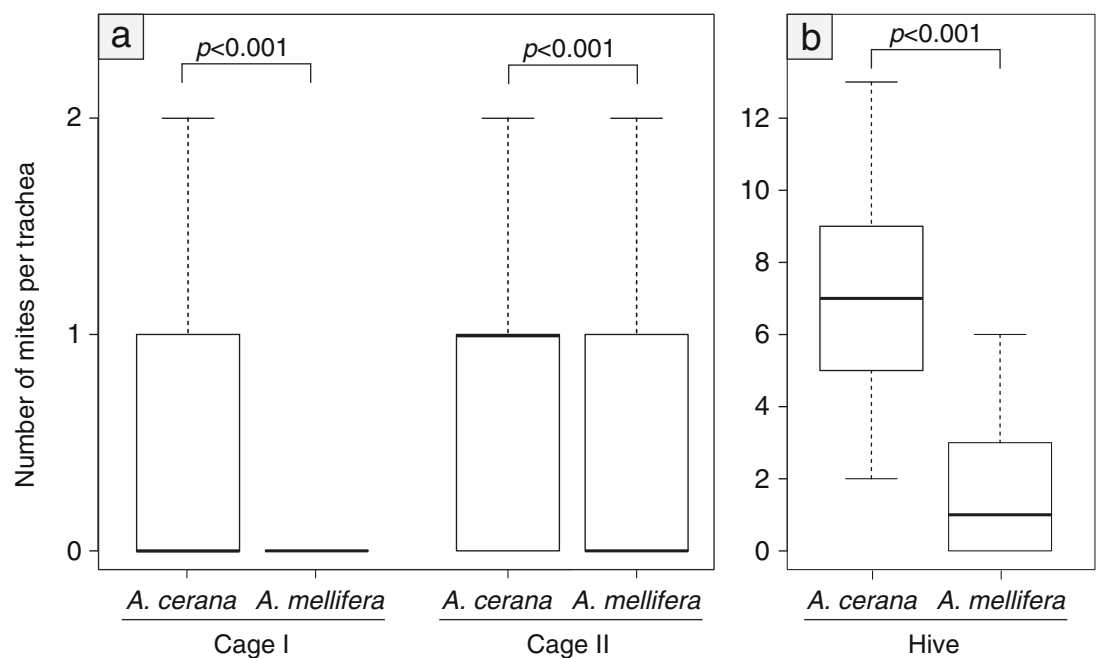

Figure 3. Number of adult mites Acarapis woodi per the large thoracic trachea in Apis cerana and Apis mellifera in inoculation experiments of cages (a) and a hive (b). Boxplots demonstrate the lower quartile, median, and upper quartile, and whiskers represent 1.5 times the interquartile range. The statistical comparisons were performed using a Mann-Whitney $U$ test. 
cage II and less than that of A. mellifera in hive inoculation assays ( $p<0.05$, chi-square test) (Table I). The mean fecundities of the cage experiments were not different between bee species ( $p>0.05, t$ test), while in the hive experiment, the mean fecundity of 3.48 in A. mellifera was significantly higher than that of 2.20 in A. cerana $(p<0.001, t$ test) (Table I). There was no significant intraspecific difference of fecundities among origins of target bees in each assay ( $p>0.05$, ANOVA) except for A. cerana in the cage II assay $(p<0.05, t$ test $)$.

\subsection{Experiment 3-interspecies comparison in frequency of grooming behaviors}

There were few records of "allogrooming" and "being allogroomed" in our tests. A. mellifera did more thorax-autogrooming per scan than did A. cerana, although the frequency of all autogrooming was higher in A. cerana than in A. mellifera (Figure 4).

\section{DISCUSSION}

Migrating female tracheal mites entered the tracheae of newly emerged bees in A. cerana twice more frequently than those in A. mellifera.

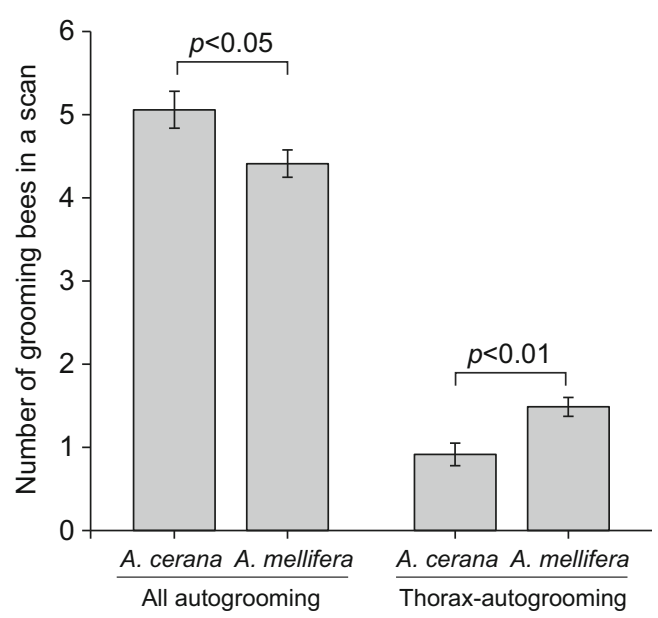

Figure 4. The frequency of "autogrooming" or "thoraxautogrooming" in Apis cerana and Apis mellifera using a visual scan $(6 \mathrm{~s} \times 10$ individuals $)$ in an observation glass cage. The statistical comparisons were performed using a Mann-Whitney $U$ test.
Considering that the mites infest younger bees more frequently in both honey bee species and that the age-related declines of infestation rate of both species were similar (experiment 1), A. cerana is more susceptible to infesting foundress mites than is A. mellifera. However, fecundities and ratios of larvae to eggs showed varying values among experiments and between species. These different values among experiments may be mainly attributed to different longevities of host bees. The differences observed between A. cerana and A. mellifera may indicate some different symptom for mite infestation between species, even though showing opposite results between the cage and hive experiments. For instance, mite fecundity in A. mellifera was 1.6 times higher than in A. cerana only in the hive inoculation assay. It is considered that the mite laid more offspring in the tracheae of A. mellifera or that the foundress mite in A. mellifera had migrated further into or out of the trachea as in the cases of Gary et al. (1989) and Mcmullan and Brown (2005). The ratio of larvae to eggs is an indicator of developmental rate because the time of entering the tracheae is similar between species as seen in experiment 1 and the distribution of migrating female mites was also not affected by whether tracheae contained mites or not (Gary and Page 1987). Therefore, we expect that the higher fecundity in A. mellifera and the variation of ratio larvae to eggs are possibly caused by different broodnest temperature (Tan et al. 2012) or some nutritional factor, and possible explanations warrant further study. In summary, we propose that more migrating mites enter the tracheae of A. cerana, and this is a primary factor causing the explosive epidemic of tracheal mites in A. cerana, while few mites are observed in A. mellifera in Japan, even though the use of Varroa treatments in A. mellifera colonies may be having some effect in tracheal-mite suppression.

The higher susceptibility to migrating mites entering into the trachea in A. cerana could help to explain the imbalance in the host-parasite relationship. One factor is surely the parasite preference to its host. When female mites migrate to new hosts, they quest and seek out the hosts using cues of specific hydrocarbons of bee's cuticle (Phelan et al. 1991). The difference in proportions of the hydrocarbons between A. cerana and A. mellifera (Lee et al. 2003) might influence 
the mite preference. Another factor is concerned with the host resistance to parasitic infestation. Previous studies in A. mellifera have shown that resistance to tracheal mites is in part due to allogrooming and autogrooming to remove the migrating mites (Danka and Villa 1998, 2003; Pettis and Pankiw 1998). Our results show that A. mellifera autogroom their thorax by using their middle legs twice as frequently as A. cerana, indicating that $A$. mellifera can more efficiently remove the migrating mites. However, we need to compare the bee's responses to the mite directly as was done by Pettis and Pankiw (1998) and Danka and Villa (2003). The lower frequency of grooming behavior is likely one cause of the higher susceptibility to the mite in A. cerana . Such a lack of a balance in the host-parasite relationship is seen in A. mellifera and Varroa mite. A. mellifera shows a higher susceptibility to the new parasite Varroa mite since A. mellifera has no effective defense to Varroa mite, while A. cerana shows a lower susceptibility since A. cerana has established effective behaviors of grooming and biting to remove Varroa mite after a long history of coevolution (Peng et al. 1987; Büchler et al. 1992). Further work is required to examine in more detail the factors affecting differential susceptibility to the tracheal mite in two honey bee species.

\section{ACKNOWLEDGMENTS}

We are grateful to Fumi Konno and Mio Nishiyama of NIES for their assistance in preparing experiments and dissecting bee samples, and to Ayumi Nakamura of NILGS and Akira Suwa and Kunihiko Numajiri in Tsukuba City for providing bee samples. We also thank Koichi Goka, Shigeki Kishi, Toshio Aoki, and our colleagues of NIES and Yoshio Suzuki, Chizuko Yoshida, Akira Kawada, and Jun Arai of the Kawakami F.C. for their kind advice and help. This study was supported by JSPS KAKENHI Grant No. 26290074, the Environment Research and Technology Development Fund (No. 5-1407) of the Ministry of the Environment, Japan, and the Sumitomo Foundation.

Différence de sensibilité à l'acarien des trachées Acarapis woodi, entre Apis cerana et Apis mellifera abeille asiatique / abeille européenne / Acari / comportement de toilettage / différence comportementale

Unterschiedliche Anfälligkeiten bei Apis cerana und Apis mellifera gegenüber der Tracheenmilbe Acarapis woodi

Asiatische Honigbienen / Europäische Honigbienen / Acarapis woodi / Anfälligkeit / Putzverhalten

\section{REFERENCES}

Anderson, D.L., Trueman, J.W.H. (2000) Varroa jacobsoni (Acari : Varroidae) is more than one species. Exp. Appl. Acarol. 24 (3), 165-189

Büchler, R., Drescher, W., Tornier, I. (1992) Grooming behavior of Apis cerana, Apis mellifera and Apis dorsata and its effect on the parasitic mites Varroa jacobsoni and Tropilaelaps clareae. Exp. Appl. Acarol.16 (4), 313-319

Bailey, L., Lee, D.C. (1959) The effect of infestation with Acarapis woodi (Rennie) on the mortality of honey bees. J. Insect Pathol. 1, 15-24

Danka, R.G. (2001) Resistance of honey bees to tracheal mites, in: Webster, T.C. and Delaplane, K.S. (Eds.), Mites of the honey bee, Dadant and Sons, Hamilton, pp. 117-129

Danka, R.G., Villa, J.D. (1998) Evidence of autogrooming as a mechanism of honey bee resistance. J. Apic. Res. 37, 39-46

Danka, R.G., Villa, J.D. (2003) Autogrooming by resistant honey bees challenged with individual tracheal mites. Apidologie 34 (6), 591-596

Eckert, J.E. (1961) Acarapis mites of the honey bee, Apis mellifera Linnaeus. J. Insect Pathol. 3, 409-425

Eischen, F.A. (1987) Overwintering performance of honey bee colonies heavily infested with Acarapis woodi (Rennie). Apidologie 18, 293-304

Eischen, F.A., Cardoso-Tamez, D., Wilson, W.T., Dietz, A. (1989) Honey production of honey bee colonies infested with Acarapis woodi (Rennie). Apidologie 20 (1), 1-8

Eischen, F.A., Pettis, J.S., Dietz, A. (1986) Prevention of Acarapis woodi; infestation in queen honey bees with Amitraz. Am. Bee J. 126 (7), 498-500

Gary, N., Page, R., Jr., Lorenzen, K. (1989) Effect of age of worker honey bees (Apis mellifera) on tracheal mite (Acarapis woodi ) infestation. Exp. Appl. Acarol. 7 (2), 153-160

Gary, N.E., Page, J.R.E. (1987) Phenotypic variation in susceptibility of honey bees, Apis mellifera, to infestation by tracheal mites, Acarapis woodi. Exp. Appl. Acarol. 3 (4), 291-305

Kojima, Y., Yoshiyama, M., Kimura, K., Kadowaki, T. (2011) PCR-based detection of a tracheal mite of the 
honey bee Acarapis woodi. J. Invertebr. Pathol. 108 (2), 135-137

Lee, C.J., Shim, J.H., Shen, J.Y., Park, S.C. (2003) Chemical analysis of cuticular hydrocarbons in Apis mellifera I. and Apis cerana F. Korean J. Appl. Entomol. 42 (1), 9-13 (in Korean)

Lee, D.C. (1963) The susceptibility of honey bees of different ages to infestation by Acarapis woodi (Rennie). J. Insect Pathol. 5, 11-15

Lorenzen, K., Gary, N.E. (1986) Modified dissection technique for diagnosis of tracheal mites (Acari, Tarsonemidae) in honey-bees (Hymenoptera, Apidae). J. Econ. Entomol. 79 (5), 1401-1403

Maeda, T. (2015) Infestation of honey bees by tracheal mites, Acarapis woodi, in Japan. J. Acarol. Soc. Jpn. 24 (1), 9-17 (in Japanese with English abstract)

Maeda, T., Sakamoto, Y. (2016) Tracheal mites, Acarapis woodi, greatly increase overwinter mortality in colonies of the Japanese honeybee, Apis cerana japonica . Apidologie: 10.1007/s13592-016-0434-x

McMullan, J.B., Brown, M.J.F. (2005) Brood pupation temperature affects the susceptibility of honeybees (Apis mellifera) to infestation by tracheal mites (Acarapis woodi). Apidologie 36, 97-105

McMullan, J.B., Brown, M.J.F. (2009) A qualitative model of mortality in honey bee (Apis mellifera) colonies infested with tracheal mites (Acarapis woodi). Exp. Appl. Acarol. 47 (3), 225-234

Ministry of Agriculture, Forestry and Fisheries. (2011) The outbreak situation of the monitoring epidemic http:// www.maff.go.jp/j/syouan/douei/kansi_densen/ kansi_densen.html.

Peng, Y.S., Fang, Y.Z., Xu, S.Y., Ge, L.S. (1987) The resistance mechanism of the Asian honey bee, Apis cerana Fabr, to an ectoparasitic mite, Varroa jacobsoni Oudemans. J. Invertebr. Pathol. 49 (1), 54-60

Pettis, J.S., Cox, R.L., Wilson, W.T. (1988) Efficacy of fluvalinate against the honey bee tracheal mite, Acarapis woodi, under laboratory conditions. Am. Bee J. 128 (12), 806

Pettis, J.S., Pankiw, T. (1998) Grooming behavior by Apis mellifera L. in the presence of Acarapis woodi (Rennie)(Acari: Tarsonemidae). Apidologie 29, 241-253

Pettis, J.S., Wilson, W.T. (1996) Life history of the honey bee tracheal mite (Acari: Tarsonemidae). Ann. Entomol. Soc. Am. 89 (3), 368-374
Pettis, J.S., Wilson, W.T., Eischen, F.A. (1992) Nocturnal dispersal by female Acarapis woodi in honey bee (Apis mellifera) colonies. Exp. Appl. Acarol. 15 (2), 99-108

Phelan, P.L., Smith, A., Needham, G. (1991) Mediation of host selection by cuticular hydrocarbons in the honeybee tracheal mite Acarapis woodi (Rennie). J. Chem. Ecol. 17 (2), 463-473

R Development Core Team. (2014) R: A language and environment for statistical computing http://www.rproject.org/.

Rennie, J. (1921) Isle of Wight disease in hive bees - acarine disease: the organism associated with the disease-Tarsonemus woodi, n. sp. Earth and Environmental Science Transactions of the Royal Society of Edinburgh 52 (4), 768-779

Ruttner, F. (1988) Biogeography and taxonomy of honeybees. Springer, Berlin, Heidelberg

Sammataro, D., Gerson, U., Needham, G. (2000) Parasitic mites of honey bees: life history, implications, and impact. Annu. Rev. Entomol. 45, 519-548

Sammataro, D., Needham, G. (1996) Host-seeking behaviour of tracheal mites (Acari: Tarsonemidae) on honey bees (Hymenoptera: Apidae). Exp. Appl. Acarol. 20 (3), 121-136

Sasaki, M. (1999) Wonders of the Japanese honeybee- biology of northernmost Apis cerana . Kaiyusha, Tokyo (in Japanese)

Scott-Dupree, C.D., Otis, G.W. (1992) The efficacy of four miticides for the control of Acarapis woodi (Rennie) in a fall treatment program. Apidologie 23 (2), 97-106

Solignac, M., Cornuet, J.M., Vautrin, D., Le Conte, Y., Anderson, D., Evans, J., Cros-Arteil, S., Navajas, M. (2005) The invasive Korea and Japan types of Varroa destructor, ectoparasitic mites of the Western honeybee (Apis mellifera), are two partly isolated clones. Proc Biol Sci 272 (1561), 411-419

Tan, K., Yang, S., Wang, Z.W., Radloff, S.E., Oldroyd, B.P. (2012) Differences in foraging and broodnest temperature in the honey bees Apis cerana and A. mellifera . Apidologie 43 (6), 618-623

Wilson, W.T., Pettis, J.S., Henderson, D.E., Morse, R.A. (1997) Tracheal mites, in: Morse, R.A. and Kim, F. (Eds.), Honey bee pests, predators, and diseases, a. I. Root Company, Ohio 